\title{
El consumo de aspirina no disminuyó la incidencia de cáncer colorrectal.
}

Aspirin Use and Colorectal Cancer: Post-Trial Follow-up Data from the Physicians ` Health Study. Stürmer T, Glynn RJ, Lee I, et al. Ann Intern Med. 1998;128:713-720

\section{Objetivo}

1.- Determinar el efecto del uso de la aspirina sobre la incidencia del cáncer colorrectal luego de 12 años en un grupo en que el consumo fue aleatorizado durante un primer periodo, y luego voluntario. 2.- Identificar los factores asociados al uso voluntario regular de la aspirina

\section{Diseño}

Estudio clínico aleatorizado y controlado (periodo inicial), y luego seguimiento prospectivo de la cohorte.

\section{Lugar \\ Estados Unidos. Realizada por la Harvard Medical School, Harvard School of Public Health, y la University of Ulm. \\ Participantes \\ Se incluyeron 22071 médicos hombres sanos de entre 40 y 84 años que en el año 1982 fueron aleatorizados para recibir $325 \mathrm{mg}$ de aspirina o un pla- cebo idéntico día por medio. Luego de una duración del estudio aleatori- zado de cinco años, los sujetos permanecieron en seguimiento, sin realizar intervención (hasta un promedio de 12 años). Durante el seguimiento pos- terior, el consumo regular de aspirina se realizó de manera voluntaria.}

\section{Evaluación de Factores de Riesgo}

Por medio de cuestionarios anuales se evaluaba sobre el uso de aspirina, y otras variables, incluída la ocurrencia de cáncer colorrectal. El resulta- do se expresó en riesgo relativo de cáncer de colon en tomadores de aspirina. Las variables potencialmente confundidoras* consideradas en el ajuste fueron edad, índice de masa corporal, uso de tabaco y alcohol.

\section{Resultados Principales}

El análisis se realizó por intención de tratar. Se diagnosticaron 341 pacientes con cáncer colorrectal. No se encontraron diferencias en la incidencia entre el grupo aspirina y el placebo luego de 12 años de seguimiento, RR 1.03 (IC95\% 0,83-1,28). El 71\% de los participantes decidieron continuar recibiendo aspirina luego de la randomización. El grupo de pacientes con ingesta voluntaria (regular e irregular) de aspirina tuvo similar incidencia de cáncer colorrectal que los que no la recibieron. Las variables independientes asociadas al uso persistente de aspirina fueron la edad, el ejercicio regular, la masa corporal, el consumo de alcohol, la hipertensión, la enfermedad coronaria y la cefalea.

\section{Conclusiones}

Tanto en el análisis aleatorizado como en el período observacional no se constató relación entre el uso de aspirina y la incidencia de cáncer colorrectal. La baja dosis de aspirina y el corto seguimiento pueden ser variables que expliquen la falta de diferencia. Sin embargo, otras características asociadas al uso de aspirina podrian explicar la diferencia reportada por estudios observacionales*.

\section{COMENTARIO}

El presente estudio es una continuación de una evaluación aleatorizada del valor de la ingesta de $325 \mathrm{mg}$ de aspirina día por médio para disminuir la incidencia de cáncer colorrectal. El mencionado estudio, denominado Physicians' Health Study ${ }^{1}$, es el único estudio aleatorizado sobre el uso de aspirina y cáncer colorrectal (aunque el objetivo primario del mismo fue evaluar la relación del consumo de aspirina y la mortalidad cardiovascular). A diferencia de la mayoría de los estudios observacionales* 2-3 no mostró un beneficio de la aspirina en cuanto la reducción del cáncer colorrectal. Lo reducido de la dosis y su corto seguimiento pudieron. ser responsables de esta falta de evidencia. Por eso, el principal objetivo del presente trabajo fue extender el seguimiento a 12 años. Además se estudiaron las características de aquellos que habían optado voluntariamente por la ingesta de aspirina luego de finalizada la aleatorización para determinar si las mismas podrían ser factores relacionados al cáncer colorrectal y por lo tanto ser fuente de sesgos durante la fase observacional. El tercer objetivo fue establecer si la ingesta adicional (voluntaria) durante los años siguientes a la finalización de la randomización producía un efecto protector más pronunciado que aquellos que la habían discontinuado.
Las conclusiones arribadas sobre la base de este estudio aportan datos para un mejor entendimiento de la relación entre la ingesta de aspirina y el cáncer colorrectal. Si bien, la dosis utilizada es baja y el seguimiento menor que el presentado en los estudios observacionales* queda claro que si el efecto protector fuera marcado, las diferencias hubieran podido ser detectadas. Por otro lado la relación encontrada entre la ingesta de aspirina y otros factores relacionados al cáncer (por ejemplo la ingesta de alcohol) le resta valor a los estudios observacionales donde estas variables no fueron controladas.

Finalmente, otro factor a tener en cuenta es la forma en la que se estimó la incidencia del cáncer colorrectal. Como en la mayoría de los estudios sobre cáncer colorrectal, el diagnóstico no fue reglado ni evaluado. Así, por ejemplo, podría especularse que tal vez la aspirina predispuso al sangrado de lesiones colónicas con el consiguiente diagnóstico más temprano o de lesiones de otra forma asintomáticas.

En resumen, creo que este trabajo es valioso ya que además de una evaluación randomizada aporta datos que ayudan a comprender mejor los estudios observacionales*.

*Ver glosario 